\title{
Aeroelastic modeling of wind loading on a cable-net supported glass wall
}

\author{
Zhifu Gu • Letian Yang • Yan Li · Bin Chen
}

Received: 2 March 2009 / Revised: 13 September 2009 / Accepted: 22 September 2009 / Published online: 9 January 2010

(C) The Author(s) 2010. This article is published with open access at Springerlink.com

\begin{abstract}
Wind loading study on a cable-net supported glass wall is conducted by means of wind tunnel tests. An equivalent aeroelastic model is designed and constructed. Response of displacements of the wall is measured and analyzed. In order to design a glass wall under wind loading, the "windvibration factor" is estimated and discussed. In fact, the mechanism of wind acting on the wall is commonly known not only as positive pressure, but also as negative pressure caused by the flow separation on the corners of the building. Due to the diffidence in the mechanism of wind acting, two typical response cases are classified. The results show that the dynamic response of the structure caused by the negative pressure is stronger than that of the positive pressure case. To determine the aerodynamic wind loading on a flexible part of structure on a building, wind tunnel study may be useful and play an important role.
\end{abstract}

Keywords Wind tunnel study - Cable-net supported glass wall $\cdot$ Wind-vibration factor $\cdot$ Aeroelastic model

\section{Introduction}

Since modern structures have become more flexible and larger in scale, many researchers in the structure design field start to pay more attention to the problem of dynamic wind loading on structures, especially that on the large-scale roofs and domes [1-4]. In these researches aiming at investigating the total response of structures, the rigid models were tested in wind tunnels to obtain the mean and dynamic pressure distributions on the surfaces of the structures, followed by

Z. Gu $\cdot$ L. Yang $\cdot$ Y. Li $\cdot$ B. Chen $(\varangle)$

LTCS and College of Engineering, Peking University,

100871 Beijing, China

e-mail: bc@pku.edu.cn the calculations of the dynamic responses of the structures using finite element programs, such as ANSYS. However, the calculated response based on the rigid model could not fully reflect the real response of the prototype. For example, the fluctuating forces induced by the motion of the structure itself when enduring the wind cannot be captured by calculating of the total responses using the rigid model.

Aeroelastic models of buildings and structures are designed to simulate the dynamic properties for the modes of vibration that contribute significantly to the wind-induced responses. Such models, representing the structure in full or in part, are capable of providing information on the mean and dynamic wind loads and the overall wind-induced responses of structures. Normally, aeroelastic models exhibit scaled elastic structural behaviors. This information is usually extrapolated analytically or by numerical methods to examine the behavior of the structure as it approaches its maximum capacity due to material failure, instability, or fatigue. However, the enhancements of aeroelastic models through mass modeling, damping, and stiffness scaling [5] can provide direct information on the responses of a structure to the wind forces. Depending on the features of the structure and the purpose of the experiments, three types of aeroelastic modeling techniques may be chosen from, which are replica, equivalent, and section models.

Nevertheless, due to the complexity of the individual structure and the additional requirements of simulation which are related to the model construction, there have been few works reported recently, especially on the equivalent model.

The New Poly Building (NPB), as one of the landmark building for China, is located in the center of Beijing. The cross-section of the building is an equilateral right triangle with the right angle side $76 \mathrm{~m}$ long, and a height of $106 \mathrm{~m}$. At its northeast elevation (face of the hypotenuse) there is a Y-shaped cable-net supported glass wall, which is much more 
flexible as compared with the main structure of the building. The proper design of the flexible structure requires determining the aerodynamic wind loading on the wall, as well as its response. Therefore, the wind loading study was carried out using wind tunnel test. Two stages of model tests: a rigid model for the static wind loading on the building at various wind directions and an aeroelastic model for the aerodynamic loading were performed. The rigid model experiment was carried out first, which provides the information of wind pressure distributions on the building, especially the cablenet wall structure at various wind directions.

This paper reports the results of aeroelastic model experiments. The emphasis of this paper is on the considerations of design and construction of the aeroelastic model, data reduction with "wind-vibration factor" analysis for engineering purpose, as well as the different mechanism of aerodynamic response of the wall under different wind conditions.

\section{Experimental apparatus and procedure}

\subsection{Wind tunnel}

The wind tunnel tests were carried out in a modified lowspeed aeronautical wind tunnel with open test section of $2.25 \mathrm{~m}$ in diameter and $3.65 \mathrm{~m}$ in length at Peking University, Beijing, China. The tunnel's testing section was modified by using a ground plate, which is lengthened through the throat of the tunnel into the stilling chamber and to a boundary layer wind tunnel. The vortex generator, which uses spires, and the barrier wall were set into the throat, and the roughness elements were provided on the ground plate. With these devices, the whole length of working section of $7.7 \mathrm{~m}$ was available to develop the suitable atmospheric boundary layer (ABL) flow. A similar example of aeronautical return flow wind tunnel with open circular test section was given by Ruscheweyh [6], which was modified to a boundary layer wind tunnel.

According to the LCDBS (GB 50009-2001) [7], a power law approximation with the exponent $\alpha=0.22$ was adopted for the mean wind velocity profile. The test models were mounted on a turntable of $2 \mathrm{~m}$ in diameter, which was located at the end of test section, allowing any wind direction to be simulated by rotating the models to the appropriate angular position in the wind tunnel.

\subsection{Models}

\subsubsection{Rigid model}

At the first stage of the investigation, a 1:300 scale rigid acrylic model of NPB was constructed to meet the requirement of geometric scale of the model and the prototype.
A total of 421 pressure taps were placed on the surface, among which 73 taps were located on the Y-shaped cablenet supported glass wall system in the northeast elevation. The models were tested together with the existing and the proposed-to-be-constructed surroundings within a radius of $300 \mathrm{~m}$.

\subsubsection{Aeroelastic model}

2.2.2.1 Structure design of the prototype The NPB is a 24 storey high composite structure consisting of reinforced concrete shear walls and steel moment frames. The northeast cable-net wall is a faceted planar system formed by folding a two-way cable-net system around two V-shaped fold lines. The fold lines consist of $200-220 \mathrm{~mm}$ diameter parallel strand cables. The overall dimension of the cable-net wall is approximately $87.8 \mathrm{~m}$ high and $57.6 \mathrm{~m}$ wide. The cablenet wall consists of horizontal cables, which predominantly resist out of plane forces due to wind or seismic loading, and vertical cables, which are predominantly used to carry the self-weight of the glass panels upwards to the base building structure. At the top of the cable-net wall, each vertical cable is connected to the base building structure through a steel spring to maintain the cable tension as the base building structure deflects due to live loadings. The northeast cablenet wall consists of a regular rectangular grid of glass panels that has been faceted by folding planes of glass around the main parallel strand cable elements. The vertical cables are spaced at $1,375 \mathrm{~mm}$ on center immediately above the lantern, and 1,233 mm on center otherwise (projected dimensions). The horizontal cables are spaced at $1,333 \mathrm{~mm}$ on center typically.

2.2.2.2 Model design and contraction For wind tunnel simulations of the aerodynamic and aeroelastic behavior of bluff bodies, the effect of turbulence characteristics of the flow and the Reynolds number effects must be considered [8]. In this study, by means of simulation ABL flow in the wind tunnel test section, the effect of turbulence characteristics of the flow was satisfied. On the other hand, the bluff bodies with sharp corners tend to cause immediate flow separation, independent of the Reynolds number of the flow. For this reason it is generally assumed that if the flow is adequately simulated, pressures on rectangular and other sharp-cornered structures can be adequately reproduced in the wind tunnel [8]. Therefore, it is expected that the Reynolds number effects would be very limited in this experiment.

According to the characteristic of the cable-net supported glass wall system, an equivalent aeroelastic model was designed and constructed, as the same geometric scale 1:300 of the rigid model. Unlike replica models, which are in complete dynamic similitude with the full-scale structure, 
Fig. 1 The model setting in the wind tunnel and the inside view of the cable-net supported wall system of the model
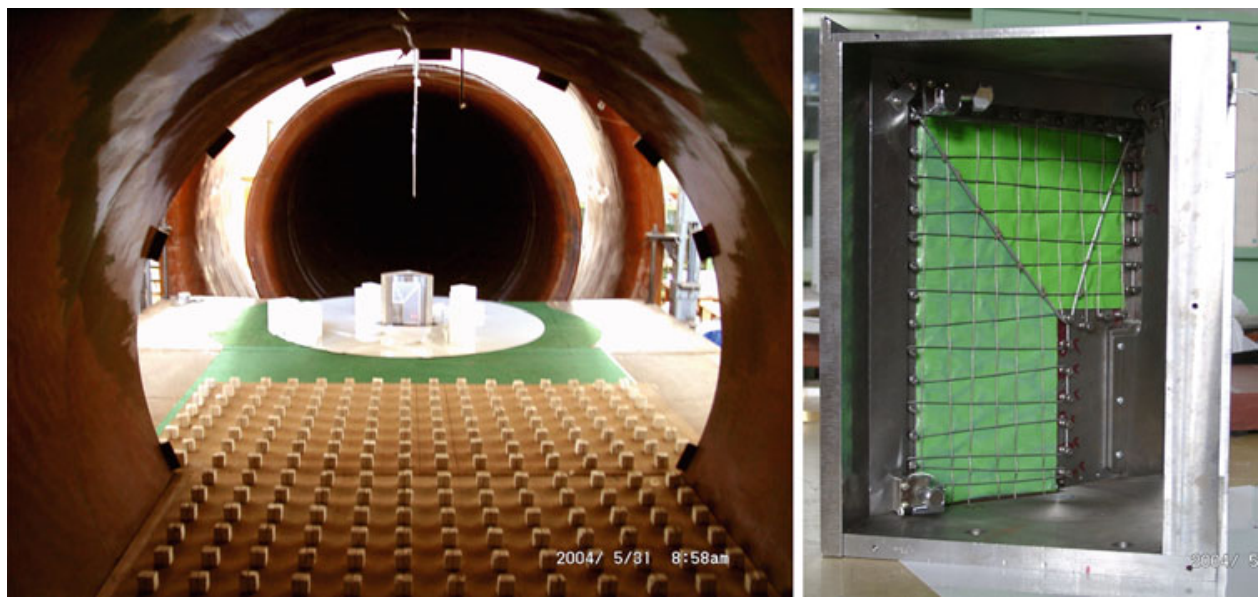

Fig. 2 The first three mode shapes of the equivalent aeroelastic model. a 1 st mode $f=220.6 ; \mathbf{b} 2$ nd mode $f=240 ; \mathbf{c} 3$ rd mode $f=292.6$
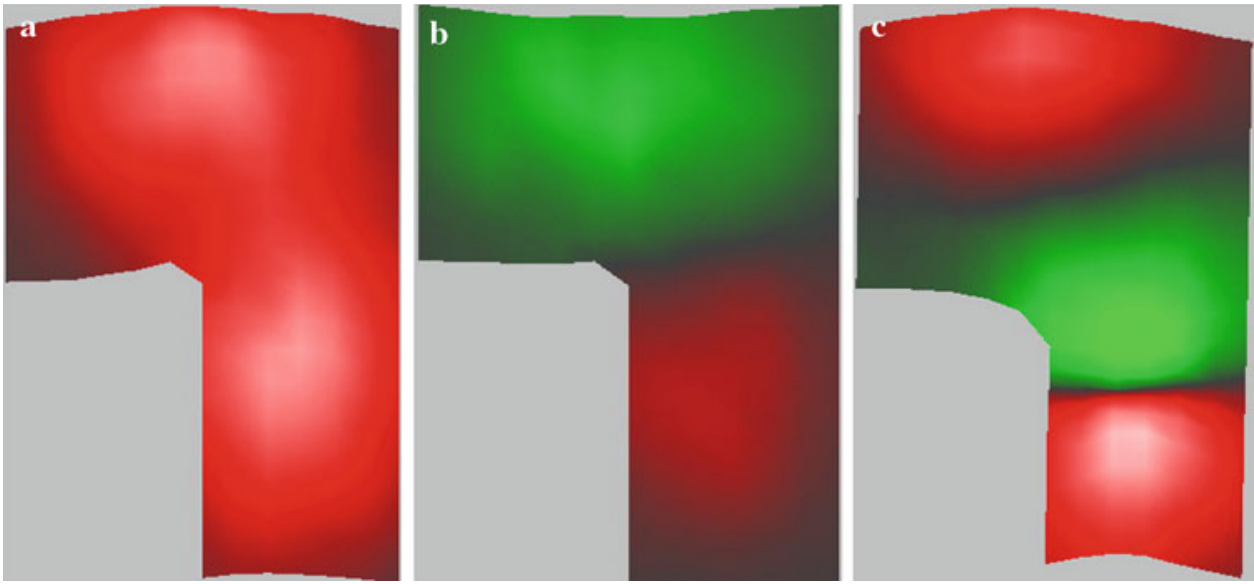

equivalent aeroelastic models are designed to simulate only particular structure behavior.

According to the NPB structure designer, the dynamic response calculations of the cable-net supported glass wall system were separated out from the rest of the building. In other words, in the calculation of the cable-net system response, the fixed boundary condition was assumed at the locations where it connects with the rigid mainframe structure of the building. According to the calculation results, the frequencies of the first three modes are 0.810, 0.912, and 0.959 , respectively, in full-scale.

The parameters of the model were reduced from the fullscale cable-net supported glass wall system, such as mass, damping, and stiffness scaling of the model to the prototype. To design the model, a structure analysis program based on finite element method was chosen to calculate the structure needed, the types of materials and the mass distribution for the scaled model. The same steel cable-net system in the prototype was also adopted in the structure of the model, so as to satisfy the similarity of damping ratio. In order to reproduce the supposed condition of the rigid mainframe structure of the building, the heavy steel was adopted to construct the other part of the building. The model was fixed on the reinforced turntable during the wind tunnel testing.

As the model was constructed based on the calculated parameters, the actual dynamic properties of the model must be verified. The parameters of real model structure such as the tension of the cable, should be adjusted to meet the goals of the model design by as much as possible, especially the first three modes of vibration and their frequencies. Several cycles of adjusting and verification may be necessary. The model setting in the wind tunnel and the inside view of the cable-net supported wall system of the model are shown in Fig. 1.

Finally, the measured shapes of the first three modes of the model agree well with that of the prototype, and their frequencies are 220.6, 240, and 296.9, respectively, as shown in Fig. 2. The frequency sample rate used in the mode adjusting and verification is $2,000 \mathrm{~Hz}$. The averaged frequency ratio of the first three modes between the model and the prototype is 274.

For a particular mode of vibration of a properly scaled aeroelastic model, the relationship between length, time, and velocity is based on the equality of the reduced frequency in 
Table 1 Relationships between wind speeds used in wind tunnel and in prototype

\begin{tabular}{lccc}
\hline $\begin{array}{l}\text { Wind speed at reference } \\
\text { height in wind tunnel }(\mathrm{m} / \mathrm{s})\end{array}$ & 15 & 18 & 20 \\
$\begin{array}{l}\text { Wind speed at } 10 \mathrm{~m} \text { height } \\
\text { in wind tunnel }(\mathrm{m} / \mathrm{s})\end{array}$ & 8.9 & 10.7 & 11.9 \\
\begin{tabular}{l} 
Full-scale at $10 \mathrm{~m}$ height $(\mathrm{m} / \mathrm{s})$ \\
\hline
\end{tabular} & 9.8 & 11.8 & 13.1 \\
\hline
\end{tabular}

model and in full scale, and can be expressed as

$\left(\frac{f_{o} L}{V}\right)_{\mathrm{M}}=\left(\frac{f_{o} L}{V}\right)_{\mathrm{P}}$,

where $f_{o}, L$, and $V$ are the natural frequency of a vibration mode, a characteristic length dimension, and a characteristic wind speed, respectively. The subscripts $\mathrm{M}$ and $\mathrm{P}$ refer to model and prototype, respectively.

Aerodynamic measurements should be carried out at several speeds, selected to simulate a representative range of the full-scale wind speed [5].

Three wind speeds: 15,18 , and $20 \mathrm{~m} / \mathrm{s}$ at the reference height were selected to find wind speed effects on the wall before experiments and the corresponding speeds at $10 \mathrm{~m}$ height in full-scale as shown in Table 1.

The results show that the variation of wind speeds only has a small effect on the normalized results of response, especially on the results of "wind-vibration factors", which will be described in detail later. It also justifies the assumption of a very limited effect due to the Reynolds number in this study. Therefore, a wind speed of $15 \mathrm{~m} / \mathrm{s}$ was adopted for all the tests.

Unfortunately, because of the limitation of the capability of the wind tunnel, the higher wind speed, approaching the maximum designed wind speed, cannot be reached.

The relationship of times between model and prototype can be expressed as follows:
$\frac{T_{\mathrm{M}}}{T_{\mathrm{P}}}=\frac{L_{\mathrm{M}} / L_{\mathrm{P}}}{V_{\mathrm{M}} / V_{\mathrm{P}}}$,

where $T$ is time.

The designed 50-year maximum $10 \mathrm{~min}$ averaged mean wind speed at $10 \mathrm{~m}$-height is $23.3 \mathrm{~m} / \mathrm{s}$ in full-scale, which is equivalent to a wind speed of $21.3 \mathrm{~m} / \mathrm{s}$ in wind tunnel testing. The requirement of sampling time of $1 \mathrm{~h}$ in full-scale is converted to a sample time of $13.1 \mathrm{~s}$ in the wind tunnel test. In experiments, a data sampling time of $35 \mathrm{~s}$ was adopted with a sampling frequency of $500 \mathrm{~Hz}$, which corresponds to a fullscale time interval of $0.5 \mathrm{~s}$ meeting the requirement of $1 \mathrm{~s}$ resolution of wind loading design on the cladding structure of a building.

\subsection{Measurement apparatus and procedure}

Due to the action of the wind, the displacement of each site on the wall was measured by the Laser Doppler Vibrometer Polytec, Controller OFV-3001, and Sensor Heads OFV-353. During the test, two channels of displacement measurement were performed at the same time. The signal of the displacement was sampled by a computer equipped with an A/D converter and the LabVIEW software.

The response of the flexible structure may dispersed at each location of different part of the structure, especially for the mode frequencies that are much closer to each other. Therefore, in order to understand the response of all surface of the wall, 18 measurement sites, P1 to P18, were assigned on the wall as shown in Fig. 3 along with the dimensions of the full-scale structure.

\section{Data reductions and results discussion}

\subsection{Data reduction}

The time-mean displacement $X$ and the root-mean-square value $\sigma_{x}$ of displacement are defined as:
Fig. 3 Locations of measurement site on the cable-net supported wall of the model together with the scales in full-scale of the structure
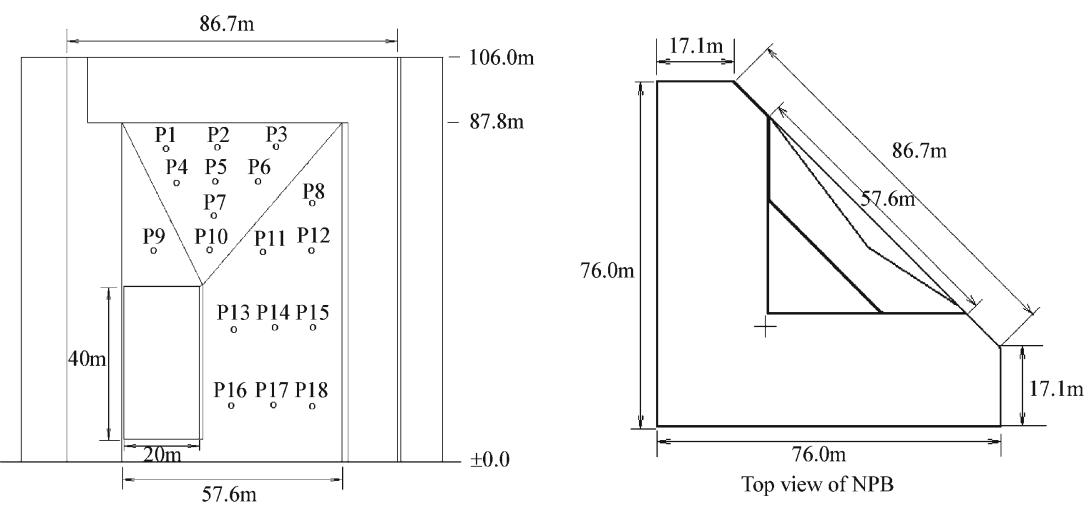
$X=\sum_{i=1}^{n} \frac{x_{i}}{n}, \quad n=500 \times 35$,

$\sigma_{x}=\sqrt{\frac{\overline{\left(x_{i}-X\right)^{2}}}{n-1}}$,

where $x_{i}$ and $n$ are the displacement and the number of sampling.

In structure wind loading design practice, the "wind-vibration factor", denoted by $\beta_{z}$, is introduced and defined as the ratio of the maximum effect of the loading to the mean effect of the loading. According to the LCDBS (GB 50009-2001), $\beta_{z}$ is given as:

$\beta_{z}=1+\frac{\xi \nu \varphi_{z}}{\mu_{z}}$

where $\xi, \nu, \varphi_{z}$, and $\mu_{z}$ are the oscillation multiple factor, the effect of fluctuation factor, the mode type factor, and the wind pressure factor as a function of heights, respectively. It is only applicable to the general cantilevered structure, such as tower-structure, chimneys and high-rise structures. Moreover, only the longitudinally response and the first mode are considered by this formula. Obviously, it is not suitable for the calculation of this project.

The aeroelastic model wind tunnel test may provide information regarding the structure response for estimating the "gust effect", other than the "wind load". Therefore the "wind-vibration factor" may be defined as the ratios of the possible maximum response to the mean response under the action of the wind. However, the concept of the load "wind-vibration factor" is different from that of the response "wind-vibration factor". Furthermore, as pointed out by some researchers [10], the definition of load "wind-vibration factor" is no longer valid in view of the nonlinear behaviors of the response of cable-net structure with the load, and the response "wind-vibration factor" may be more suitable.

The response, or displacement "wind-vibration factor", $\beta_{z}$, at any point in the structure is:

$\beta_{z}=1+\frac{\mu \sigma_{x}}{X}$

where $\mu, \sigma_{x}$ and $X$ are the oscillation multiple factor, the "root-mean-square" (RMS) of displacement, and the mean displacement, respectively.

On the other hand, the results of a research on a long-span single-layer spherical reticulated shell show that by using the displacement "wind-vibration factor" instead of the load "wind-vibration factor", the calculated results of the inertial force are almost the same [3]. Therefore, in this paper, the "wind-vibration factor" always refers to the displacement "wind-vibration factor".

As an elastic structure, the displacement also occurs in the whole NPB when subjected to the wind action. The displacement response of the whole NPB is much smaller as compared with cable-net supported wall, which is much more flexible. Although the foundation of the model is strengthened to maximum possible extent during the test, the measurement shows that the displacement of the rigid frame still needs to be considered as compared with the data from the wall. Whereas, the RMS value of the mainframe is shown to be much smaller as compared with the result of the wall. The measured mean displacement from the wall may be considered as a linear superposition of the mainframe and the wall during the test. However, this may not be the case for the RMS. The net mean displacements of the wall should be the difference between the measured mean displacement from the wall and that of the mainframe structure giving a modified expression for the conservative "wind-vibration factor" $\beta_{z m}$ :

$\beta_{z m}=1+\frac{\mu \sigma_{x}}{X-X_{0}}$,

where $X_{0}$ is the mean displacement of the mainframe.

On the other hand, as the full-scale structure, the mass and stiffness of the mainframe are much bigger, stronger and heavier than that of the cable-net system, it is reasonable to assume the mainframe to be rigid boundary condition in the calculation of the cable-net dynamic response. For the same reason, the results of modified "wind-vibration factor" $\beta_{z m}$ may provide closer estimations for the designer.

The results of first stage rigid model experiment show that the wind direction has great influence on the wind loading on the structure. Consequently, it is expected that the response of structure will also dependent sensitively on the wind direction.

In order to obtain the extreme conditions of wind-induced vibration as the wall faces to the wind and as the wall locates in the side or leeward, two typical wind directions were selected. Based on the results of the first stage rigid model study, two wind directions of $\beta=40^{\circ}$, and $\beta=290^{\circ}$ were selected, corresponding to the maximum mean positive and negative pressure load on the wall. The averaged mean pressure coefficient distributions on the wall are +0.6 and -0.4 in the wind directions $\beta=40^{\circ}$ and $\beta=290^{\circ}$, respectively.

\subsection{Results and discussion}

As indicated in Ref. [9], the dynamic response includes the action of (a) random wind gusts acting for short durations over all or part of the structure, (b) fluctuating pressures induced by the wake of the structure, including "vortex shedding forces", and (c) fluctuating forces induced by the motion of the structure itself through the wind. They may act longitudinally, laterally or torsionally, and may be further amplified by the resonance of the structure at one or more of its natural frequencies. All structures are affected to some degree by these forces. The total response may be considered as a superposition of a "background component", which acts 
Fig. 4 Time histories of displacements on site P14 at two wind directions in $1 \mathrm{~s}$.

a $\beta=40^{\circ} ; \mathbf{b} \beta=290^{\circ}$
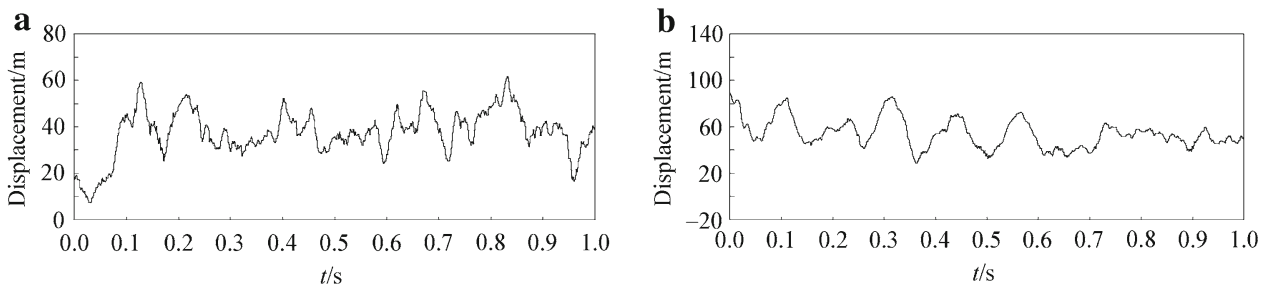

quasi-statically without any structural dynamic magnification, and a "resonant" component due to the excitation close to a natural frequency. Therefore, it is clear that the amount of the "resonant" components may be quite different depending on the features of the wind. In other words, the "wind-vibration factor" should be quite different with the mechanism of wind loading.

As the cable-net supported wall faces to the wind, the wind-induced vibrations are mainly caused by the turbulence of oncoming flow; or if the upstream structures exist, caused by the wake turbulence of upstream building. Whereas the cable-net supported wall locates at the side, as the wind passes or leeward of wind, the negative fluctuating pressure is experienced, which is usually caused by the shear layer flow separation from the edge of the structure, or the vortex shedding on the structure itself. It is evident that the density of fluctuating in flow under the separation shear layer or in the wake with vortex shedding should be much stronger than that in the oncoming flow. Therefore, the difference of mechanism in pressure fluctuating would causes differ-

Table 2 Mean and RMS values of displacement and calculated results of $\beta_{z}$ and $\beta_{z m}$ at $\beta=40^{\circ}$

\begin{tabular}{lcccc}
\hline Site & $X$ & $\sigma_{x}$ & $\beta_{z}$ & $\beta_{z m}$ \\
\hline 1 & 77.9 & 12.2 & 1.55 & 1.79 \\
2 & 88.7 & 14.2 & 1.56 & 1.76 \\
3 & 144 & 19.2 & 1.47 & 1.56 \\
4 & 75.6 & 14.1 & 1.65 & 1.95 \\
5 & 124 & 17 & 1.48 & 1.59 \\
6 & 139 & 16.5 & 1.42 & 1.50 \\
7 & 90.2 & 13.2 & 1.51 & 1.69 \\
8 & 64.7 & 10.6 & 1.57 & 1.90 \\
9 & 45.2 & 9.6 & 1.74 & 2.56 \\
10 & 62.1 & 10.6 & 1.60 & 1.97 \\
11 & 95.7 & 13.6 & 1.50 & 1.66 \\
12 & 68.6 & 9.9 & 1.51 & 1.77 \\
13 & 148.5 & 17.3 & 1.41 & 1.49 \\
14 & 177.2 & 20.4 & 1.40 & 1.47 \\
15 & 86 & 11.2 & 1.46 & 1.63 \\
16 & 104.7 & 12.2 & 1.41 & 1.53 \\
17 & 183.6 & 18.9 & 1.36 & 1.41 \\
18 & 82.8 & 10.7 & 1.45 & 1.63 \\
\hline
\end{tabular}

ent responses of the structure, so does the values of "windvibration factor", which reflects the strength of fluctuating in pressure together with the resonant vibration effect of the structure. The results of the two wind directions $\left(\beta=40^{\circ}\right.$, and $290^{\circ}$ ) justly reflect the two different features of response of an aeroelastic model due to the different mechanisms in wind action.

The displacement signals on site P14 varying with time duration of $1 \mathrm{~s}$ in wind directions of $\beta=40^{\circ}$, and $290^{\circ}$ are shown in Fig. 4. It is shown that the features of the two curves are quite different for the difference mechanism in wind actions. Besides the difference in the magnitude of displacement, there is much more fluctuating in the curve corresponding to the positive pressure compared with that of the negative pressure.

The measured mean and RMS values of displacement, in units of $\mu \mathrm{m}$, at $\beta=40^{\circ}$, and $\beta=290^{\circ}$, and the calculated results of $\beta_{z}$ and $\beta_{z m}$, are listed in Tables 2 and 3 , respectively.

The distributions of $\beta_{z}$ and/or $\beta_{z m}$ on the surface of the wall are rather smooth and uniform. It agrees with that

Table 3 Mean and RMS values of displacement and calculated results of $\beta_{z}$ and $\beta_{z m}$ at $\beta=290^{\circ}$

\begin{tabular}{lllll}
\hline Site & $X$ & $\sigma_{x}$ & $\beta_{z}$ & $\beta_{z m}$ \\
\hline 1 & 26 & 5.3 & 1.71 & 2.37 \\
2 & 26.7 & 5.8 & 1.76 & 2.43 \\
3 & 27.7 & 5.7 & 1.72 & 2.31 \\
4 & 27.7 & 6.5 & 1.82 & 2.50 \\
5 & 27.5 & 7 & 1.89 & 2.63 \\
6 & 32.2 & 7.3 & 1.79 & 2.30 \\
7 & 30.5 & 7.2 & 1.83 & 2.40 \\
8 & 27.8 & 5.1 & 1.64 & 2.17 \\
9 & 16.7 & 3.9 & 1.82 & 4.25 \\
10 & 27.5 & 5.6 & 1.71 & 2.31 \\
11 & 33.8 & 6.4 & 1.66 & 2.05 \\
12 & 32.4 & 6.2 & 1.67 & 2.09 \\
13 & 28.7 & 6.5 & 1.79 & 2.40 \\
14 & 33.9 & 8.2 & 1.85 & 2.34 \\
15 & 31.3 & 7.2 & 1.81 & 2.34 \\
16 & 23.4 & 5.8 & 1.87 & 2.86 \\
17 & 25.3 & 7.2 & 2.00 & 2.97 \\
18 & 25.6 & 5.2 & 1.71 & 2.39 \\
\hline & & & &
\end{tabular}


Fig. 5 Mean displacement distributions on the cable-net wall at wind directions of a $\beta=40^{\circ}$ and $\mathbf{b} \beta=290^{\circ}$

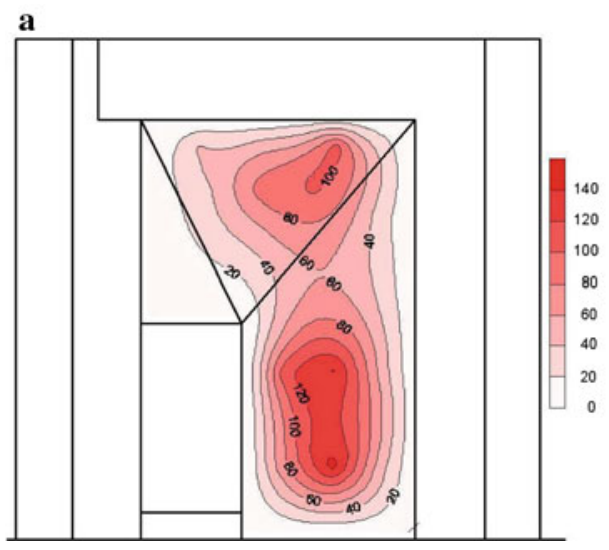

b

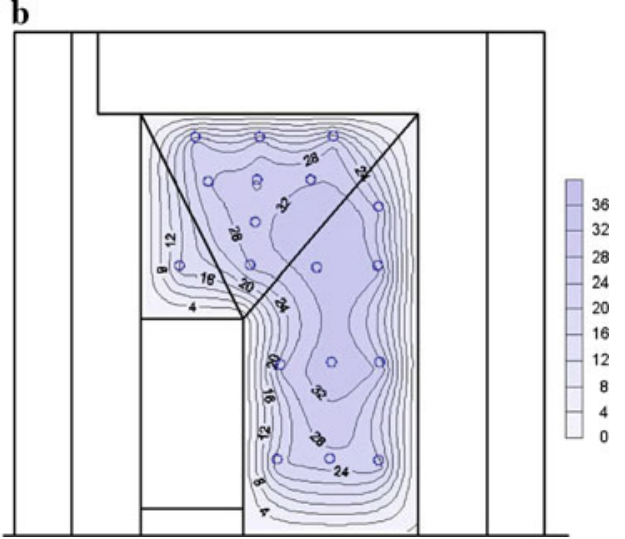

obtained for a long-span single-layer spherical reticulated shell [1].

For the positive pressure acting on the wall $\left(\beta=40^{\circ}\right)$, the averaged value $\beta_{z}$ and the modified value $\beta_{z m}$ on all 18 sites are 1.50 and 1.72 , respectively. Whereas for the negative pressure acting on the wall $\left(\beta=290^{\circ}\right)$, the averaged values of $\beta_{z}$ and the modified values of $\beta_{z m}$ on all 18 sites are 1.78 and 2.51 , respectively. In this case, the averaged value $\beta_{z}$ and the modified value $\beta_{z m}$ in the negative condition are 19 and $46 \%$ bigger than that in the positive condition, respectively, which contrasts sharply with that of the mean positive and negative pressure loading.

The mean displacement distributions on the wall under the wind action of positive $\left(\beta=40^{\circ}\right)$ and negative pressure $\left(\beta=290^{\circ}\right)$ are shown in Fig. 5. There are two maximum mean displacement regions on the wall under the positive pressure as a result of the action of the main cable located at the back of the wall. However, due to the weak connection between the wall and the main cable, as the negative pressure is acting, only one maximum mean displacement region occurs under the negative pressure.

\section{Conclusions}

The dynamics wind loading on a specially designed structure of cable-net supported wall is studied by means of an aeroelastic model wind tunnel test. The additional requirements of simulation are considered and an equivalent aeroelastic model is designed and constructed.

Results show that due to the diffidence in the mechanism of wind action on the wall, two typical cases can be classified, i.e., (1) as the wind acting on the wall directly with the positive pressure, and (2) as the flow separation from the corner of the building causing the negative pressure on the wall. Consequently, the responses of the wall are quite different due to the difference in mechanism of wind action, and the values of "wind-vibration factor", which are applied in engineering practice, are also quite different. In general, the negative pressure, which is associated with the shear layer separation and vortex shedding, induces a stronger dynamic response of the structure than the positive pressure, which is usually associated with only the turbulence in oncoming flow.

The wind tunnel study is a powerful method to determine the dynamic wind loading on a complex flexible part of a building structure.

Open Access This article is distributed under the terms of the Creative Commons Attribution Noncommercial License which permits any noncommercial use, distribution, and reproduction in any medium, provided the original author(s) and source are credited.

\section{References}

1. Li, Q.X., Lou, W.J., Yang, S.C., Sun, B.N.: Wind load factor and parametric analysis of long-span single-layer spherical reticulated shell. J. Build. Struct. 27(4), 65-72 (in Chinese) (2006)

2. Tian, Y.J., Yang, Q.S., et al.: Gust factors for large-span roof of the National Stadium. J. Build. Struct. 28(2), 26-31 (in Chinese) (2007)

3. Ding, Y., Qi, L., Zhao, Y.C., Zhu, L.P.: Wind vibration coefficient of long-span spatial structures with self-oscillating of roof and fluid-structure coupling effect. J. Build. Struct. 29(5), 101-106 (in Chinese) (2008)

4. Han, Q.H., Chen, Y., Zeng, Q.M., Liu, X.L.: Analysis of windinduced vibration coefficient for long-span reticulated dome. J. Earthq. Eng. Eng. Vib. 27(1), 38-45 (in Chinese) (2007)

5. American Society of Civil Engineers (ASCE).: Wind tunnel studies of buildings and structures. In: ASCE Manuals and Reports on Engineering Practice No. 67 (1999)

6. Ruschewyh, H.: Dynamic response of high rise building under wind action with interference effects from surrounding buildings of similar size. In: Proceedings of the Fifth International Conference, Fort Collins, Colorado, USA, pp. 725-734, July 1979

7. Load Code for the Design of Building Structures (GB 50009-2001, 2002-01). Building Industrial Press, Beijing, 2006 version

8. Simiu, E., Scanlan, R.H.: Wind Effects on Structures, 3rd edn. Wiley, New York (1996)

9. Supplement to the National Building Code of Canada.: First revisions and errata, Associate Committee on the National Building Code, National Research Council of Canada, Ottawa, January 1991

10. Wu, Y., et al.: Analysis of wind induced vibration on the support structure of Cuangzhou Airport Terminal Building. In: Proceedings of 10th National Conference on Structure Wind Engineering, Guilin, Longsheng, Nov 2001 (in Chinese) 